\title{
Consumo alimentar e excesso de peso de adolescentes de Piracicaba, São Paulo
}

\author{
Food consumption and overweight in adolescents \\ from Piracicaba, São Paulo, Brazil
}

Natacha TORAL ${ }^{1}$

Betzabeth SLATER ${ }^{1}$

Marina Vieira da SILVA²

\section{RE S U M O}

\section{Objetivo}

Este estudo avaliou o consumo alimentar e a prevalência de excesso de peso entre adolescentes de Piracicaba, São Paulo.

\section{Métodos}

Estudo observacional transversal com amostra representativa de adolescentes maiores de 10 anos de 11 escolas públicas de Piracicaba. Realizou-se auto-avaliação da maturação sexual por planilhas dos estágios de Tanner. Pelo Questionário de Freqüência Alimentar Semi-Quantitativo, investigou-se o consumo de energia, macronutrientes, frutas, hortaliças e doces. Foram aferidos peso e altura para avaliação do perfil antropométrico pelos percentis de Índice de Massa Corporal e índice de altura-para-idade.

\section{Resultados}

Foram avaliados 390 adolescentes, com média de idade de 12,4 anos, sendo 46,4\% meninos e 78,7\% púberes. O consumo médio de energia foi de $3.645 \mathrm{kcal}$ (56,2\%, 12,8\% e 33,5\% provenientes de carboidratos, proteínas e lipídios, respectivamente). Observou-se que $77,9 \%$ da amostra ingeria alto teor de gordura. $\mathrm{O}$ consumo médio de frutas, hortaliças e doces foi de 2,3, 2,4 e 4,5 porções diárias, respectivamente. Foi encontrado $21,0 \%$ de excesso de peso, $4,4 \%$ de baixo peso e 1,8\% de déficit de altura-para-idade.

\section{Conclusão}

Expressivo percentual de adolescentes apresentava reduzido consumo de frutas e hortaliças e consumo lipídico e de doces acima do recomendado, podendo haver relação com o elevado número de participantes com excesso de peso. Adolescentes devem ser alvo de programas que enfatizem a adoção de alimentação saudável, para diminuir risco e incidência de obesidade e prevenir futuros agravos à saúde.

Termos de indexação: adolescente; antropometria; consumo de alimentos; sobrepeso.

\footnotetext{
${ }^{1}$ Universidade de São Paulo, Faculdade de Saúde Pública, Departamento de Nutrição. Av. Dr. Arnaldo, 715, Cerqueira César, 01246-904, São Paulo, SP, Brasil. Correspondência para/Correspondence to: N. TORAL. E-mail: <natytb@usp.br>.

2 Universidade de São Paulo, Escola Superior de Agricultura Luiz de Queiroz, Departamento de Agroindústria, Alimentos e Nutrição. Piracicaba, SP, Brasil.
} 
450 | N. TORAL et al.

\section{A B S T R A C T}

\section{Objective}

This study assessed food consumption and prevalence of overweight among adolescents from Piracicaba, São Paulo, Brazil.

\section{Methods}

This was a cross-sectional with a representative sample of adolescents older than 10 years of 11 public schools of Piracicaba. The maturation stage of the adolescents was determined by self-examination using Tanner images. Intakes of energy, macronutrients, fruits, vegetables and sweets were determined with a semiquantitative food frequency questionnaire. Weight and height were measured to determine body mass index and height-for-age percentiles.

\section{Results}

Three-hundred ninety adolescents with a mean age of 12.4 years, $46.4 \%$ males and $78.7 \%$ pubescents were assessed. Mean energy intake was 3,645 kcal (56.2\%, $12.8 \%$ and 33.5\% of carbohydrates, proteins and fats respectively). Most of the adolescents (77.9\%) had excessive intake of dietary fat. The mean consumption of fruits, vegetables and sweets was 2.3, 2.4 and 4.5 portions per day, respectively. Twenty-one percent of the adolescents had overweight, $4.4 \%$ were underweight and $1.8 \%$ were stunted.

\section{Conclusion}

A high percentage of adolescents consumed too little fruits and vegetables and too much lipids and sweets (above the recommended intakes), which may explain why many children had overweight. Programs that promote healthy eating should also target adolescents to reduce the risk and incidence of obesity and prevent future health problems.

Indexing terms: adolescent; anthropometry; food consumption; overweight.

\section{N T R O D U ÇÃ O}

A adolescência corresponde a um estágio de vida no qual ocorrem complexas transformações somáticas, psicológicas e sociais no indivíduo. Crescer e se alimentar implicam no estabelecimento de relações, na realização de escolhas, na identificação com valores sociais e padrões estabelecidos, na adoção de diversos hábitos, horários e estilos de vida' ${ }^{1}$. Sabe-se que as práticas alimentares inadequadas são cada vez mais freqüentes nessa população. Atualmente, o padrão alimentar característico do adolescente inclui o consumo excessivo de refrigerantes, açúcares e junk foods, a reduzida ingestão de frutas e hortaliças, a adoção de dietas monótonas ou modismos alimentares, bem como a não realização do café da manhãa-4. Tais desequilíbrios alimentares favorecem a ocorrência de desvios nutricionais, além de uma ingestão insuficiente de micronutrientes ${ }^{2}$.

As práticas alimentares inadequadas e o aumento do sedentarismo entre adolescentes estão relacionados ao incremento da prevalência de obesidade, que acompanha o processo de transição nutricional, constatado nas sociedades modernas de diversos países, inclusive no Brasil ${ }^{5}$. Nas últimas décadas, tem sido verificado um aumento alarmante das taxas de excesso de peso em adolescentes brasileiros ${ }^{6}$, comparando os dados do Estudo Nacional de Despesa Familiar (ENDEF), realizado em 1974-1975, e da Pesquisa sobre Padrões de Vida (PPV), de 1996-1997. O excesso de peso entre os meninos aumentou de $2,6 \%$ para $11,8 \%$; entre as meninas, a prevalência passou de $5,8 \%$ para $15,3 \%$ nesse período.

A ocorrência da obesidade na infância e adolescência tem recebido notório destaque, devido à gravidade da manutenção dessa doença na vida adulta. A concomitância de fatores de risco durante o período de crescimento, como dislipidemias e resistência insulínica, está associada a um aumento da mortalidade entre os adultos. Tal fato destaca a importância da detecção precoce do problema, devendo ser 
analisadas as variações ponderais desde a infância, como prevenção e controle da permanência da obesidade em estágios de vida futuros ${ }^{4}$.

Entre as principais estratégias para a contenção da epidemia de obesidade entre adolescentes incluem-se a promoção de um estilo de vida ativo e o estímulo à adoção de práticas alimentares saudáveis, promovendo maior consumo de frutas e hortaliças, restringindo a ingestão de alimentos de alta densidade energética ou pobres em nutrientes, e fornecendo as informações necessárias para que os próprios adolescentes sejam capazes de realizar escolhas alimentares saudáveis ${ }^{7}$.

Para tanto, é essencial conhecer previamente as práticas alimentares e a prevalência de desvios nutricionais da população adolescente. Nesse contexto, o objetivo deste estudo foi avaliar o consumo alimentar e a prevalência de excesso de peso entre adolescentes de escolas públicas de Piracicaba, São Paulo.

\section{MÉ T O D OS}

Foram incluídos na pesquisa adolescentes de ambos os sexos, matriculados em escolas da rede pública de ensino da cidade de Piracicaba, com idade mínima de 10 anos. As entrevistas, obtidas durante o mês de novembro de 2004, foram realizadas em espaço reservado em cada escola, durante a jornada de aula.

De acordo com o último censo do Instituto Brasileiro de Geografia e Estatística, o município de Piracicaba apresentava uma população de 329.158 habitantes $^{8}$. De acordo com informações fornecidas pela Secretaria de Estado da Educação, encontravam-se matriculados 40.550 alunos em Piracicaba no ano de 2003, de todas as faixas etárias.

Para o cálculo da amostra, foram consideradas as seguintes informações: prevalência de sobrepeso em população semelhante (15\%); Odds ratio na população $(1,6)$; erro tipo I de $5 \%$ e erro tipo II de $20 \%$. O procedimento de amostragem foi do tipo aleatório simples, estratificado por séries, sendo realizado em duas etapas. $\mathrm{Na}$ primeira etapa, as unidades primárias de amostragem, que correspondem às escolas do município, foram ordenadas segundo as regiões. 0 município de Piracicaba apresentava 70 escolas públicas de ensino, localizadas em sete regiões, incluindo duas de zona rural. Dessas, 11 escolas foram sorteadas para a seleção de uma amostra representativa de adolescentes, sendo 10 de área urbana e 1 da zona rural, e havendo, no mínimo, uma escola por região do município.

Na segunda etapa da amostragem, foram ordenadas as unidades secundárias, que correspondem às classes (séries) das escolas. Em cada unidade primária de amostragem, sortearam-se as classes por amostragem aleatória simples novamente. De cada classe selecionada, realizou-se um sorteio de determinado número de alunos, de forma proporcional ao porte da escola. Desse modo, o número de unidades secundárias por unidades primárias de amostragem variou de uma até cinco. Na existência de mais de uma turma por classe, foi conduzido outro sorteio entre todas as turmas, selecionando-se quais participariam do estudo. O convite para a participação foi realizado para todos os alunos da turma sorteada, sendo entrevistados aqueles que tiveram a autorização dos pais. A partir do processo de amostragem descrito, foi determinado que o universo amostral estimado deveria ser constituído por 420 adolescentes. Realizou-se o pré-teste do presente estudo em uma escola da rede pública de ensino de Piracicaba, a qual foi excluída do processo de amostragem do estudo principal.

Para avaliação das condições socioeconômicas, um questionário contendo perguntas sobre a renda e a escolaridade foi entregue a cada adolescente, para ser respondido pelos pais ou responsáveis do mesmo e devolvido na própria escola à direção.

Coletaram-se informações do participante relativas ao nome completo, sexo e idade em 
anos. Também foi realizada uma avaliação da maturação sexual, por meio da utilização de planilhas demonstrativas dos estágios de Tanner ${ }^{10}$ em desenhos. Considerando que o presente estudo incluiu participantes na fase inicial da adolescência, adotou-se a classificação proposta pela Organização Mundial da Saúde ${ }^{11}$ para agrupar os participantes entre pré-púberes e púberes. Para tanto, foram utilizados somente os dados relativos ao desenvolvimento de mamas no sexo feminino e da genitália no sexo masculino.

A avaliação do consumo habitual foi realizada por meio da aplicação do Questionário de Freqüência Alimentar para Adolescentes (QFAA), validado por Slater et al. ${ }^{12}$. Trata-se de um questionário de freqüência alimentar semi-quantitativo, contendo 94 alimentos e 7 opções de freqüência de consumo. Para este estudo, foram utilizados dados referentes à distribuição de macronutrientes na dieta e ao consumo em porções diárias de frutas, hortaliças e doces. Dessa forma, realizou-se uma classificação de parte dos itens do QFAA entre tais grupos alimentares. Em caráter descritivo, foi avaliado também o consumo energético entre os participantes.

Com o intuito de avaliar o consumo alimentar dos grupos estudados, foram consideradas as recomendações propostas pelo "Guia alimentar para a população brasileira: promovendo a alimentação saudável" 13 , material elaborado pelo Ministério da Saúde. Foram selecionadas as recomendações da Diretriz 1 em relação à participação dos macronutrientes no valor energético total (VET) da alimentação: $55 \%$ a $75 \%$ do VET de carboidratos totais, $10 \%$ a $15 \%$ do VET de proteínas e $15 \%$ a $30 \%$ do VET de gorduras. Também foram selecionadas as recomendações da Diretriz 3 quanto ao consumo de frutas e hortaliças, que correspondem a 3 porções diárias de cada grupo alimentar, e da Diretriz 6 quanto ao consumo de açúcares e doces, cujo consumo máximo diário deve ser de 1 porção.

Para avaliação do peso corporal, os adolescentes utilizaram roupas leves e estavam descalços, sendo assim posicionados sobre uma balança eletrônica do tipo plataforma, com capacidade para $150 \mathrm{~kg}$ e sensibilidade de 100 gramas. Para a aferição da altura, foi utilizado um estadiômetro com escala em milímetros, que foi fixado em um suporte de madeira, confeccionado para o estudo com a finalidade de manter um ângulo reto entre o piso e a parede. Nessa medição, os indivíduos mantinham os pés juntos, calcanhares encostados na parede, em postura ereta, com olhar fixo no horizonte, sem fletir ou estender a cabeça. Em seguida, posicionou-se a barra horizontal do estadiômetro até mantê-la apoiada sobre a cabeça, sendo então efetuada a leitura da altura em centímetros.

Tanto o peso como a altura foram aferidos em duplicata, admitindo-se um valor máximo de diferença de $1,0 \mathrm{~cm}$ entre ambas as medidas. Foi adotado o valor médio das duas medidas para o cálculo do índice de massa corporal (IMC), definido como a relação entre o peso em quilogramas e a altura em metros elevada ao quadrado $\left(\mathrm{kg} / \mathrm{m}^{2}\right)$. A classificação do perfil antropométrico dos participantes foi realizada pelos percentis de IMC para a idade11, a partir das curvas de referência do Centers for Disease Control and Prevention ${ }^{14}$. Caracterizaram-se como de baixo peso os adolescentes com IMC inferior ao percentil 5; eutróficos, aqueles com IMC entre os percentis 5 a 84,9; com sobrepeso, os participantes com IMC entre os percentis 85 e 94,9 e obesos aqueles com IMC igual ou superior ao percentil 95. A categoria de excesso de peso foi atribuída aos casos de sobrepeso e obesidade em conjunto.

Dada a importância de identificar o crescimento linear durante a adolescência, foi avaliado também o índice altura-para-idade entre os participantes. O diagnóstico de déficit foi adotado quando a altura do adolescente estava aquém de 2 desvios-padrão da média esperada para idade e sexo, segundo a distribuição de referência do National Center for Health Statistics ${ }^{15}$. Destaca-se que a proposta do National Center for Health Statistics $^{15}$ não apresenta dados de IMC para a população, o que justificou a inclusão de um segundo sistema de referência, o do Centers for 
Disease Control and Prevention ${ }^{14}$. Posteriormente, foi avaliada a presença de associação do consumo alimentar considerado saudável, para os critérios descritos anteriormente, com a ausência de excesso de peso na população estudada.

Os dados do presente estudo, com exceção daqueles relativos ao consumo alimentar, foram digitados no programa de computador Epi Data 3.02 (The EpiData Association, Odense Denmark, 2002). Para a análise da composição nutricional, adotou-se o software Dietsys 4.01 (National Cancer Institute, USA, 1999). Foram excluídos os indivíduos que apresentavam consumo diário inferior a 5 itens do QFAA ou superior a 51 itens do QFAA e aqueles cujo consumo energético era inferior a $500 \mathrm{kcal}$ ou superior a $7000 \mathrm{kcal}$. A identificação do percentil de IMC dos adolescentes realizou-se pelo software Epi Info 3.3. Os participantes que apresentassem dados incompletos em relação a qualquer uma das variáveis do estudo foram eliminados das análises.

Após a realização do teste de Kolmogorov-Smirnov, optou-se por testes paramétricos, com a transformação das variáveis para a forma logarítmica (com exceção da referente ao percentual de macronutrientes da dieta). Para as variáveis relativas ao consumo de frutas e de hortaliças, realizou-se a transformação para logaritmo natural adicionando-se uma unidade ao dado original (equação: $z=\ln (x+1)$, sendo $x$ o número de porções consumidas), tendo em vista a existência de dados de consumo iguais a zero. Foram calculadas medidas de tendência central e de dispersão, além de comparar médias por meio dos testes de " $t$ " de Student e avaliar a associação entre variáveis com o teste do Qui-Quadrado. Adotou-se o grau de significância estatística de $5 \%$. Para a análise estatística foi adotado o software Statistical Package for the Social Sciences 10.0. Os resultados serão apresentados como médias sem transformação logarítmica para facilitar a compreensão dos resultados obtidos.

Este trabalho foi aprovado pelo Comitê de Ética em Pesquisa da Faculdade de Saúde Pública da Universidade de São Paulo (Protocolos n 848 e $n^{\circ} 1191$ ).

\section{RESULTADOS}

Foram entrevistados 431 adolescentes, número superior ao universo amostral estimado, considerando que participaram todos os alunos das turmas sorteadas que aceitaram o convite e apresentaram o consentimento dos pais. Do total, foram excluídos 41 participantes, por terem referido consumo diário inferior a 5 itens do QFAA $(n=2)$ e por terem referido consumo diário superior a 51 itens do QFAA $(n=7)$, por apresentarem consumo superior a $7000 \mathrm{kcal}(\mathrm{n}=23)$ ou, ainda, por registrarem dados incompletos $(n=9)$. A amostra final envolveu 390 participantes.

Verificou-se que uma elevada parcela dos pais ou responsáveis pelos adolescentes da amostra não soube responder, ou se recusou a fornecer informações socioeconômicas: 44,9\% e $39,2 \%$ não responderam ou não souberam responder sobre a escolaridade do pai (ou responsável do sexo masculino) e da mãe (ou responsável do sexo feminino), respectivamente, e $46,1 \%$ não responderam ou se recusaram a responder sobre a renda familiar. Foi constatado que, em média, $57,2 \%$ dos pais ou responsáveis de ambos os sexos, que responderam ao questionário, relataram ter cursado o $1^{\circ}$ grau, completo ou não. Quanto à renda, observou-se que $83,8 \%$ das famílias entrevistadas dispunham de até 5 salários mínimos por mês. A renda familiar variou de 0,1 a 11,7 salários mínimos e a média (e intervalo de confiança - IC - de 95,0\%) apresentada foi de 3,2 (IC: 2,9-3,5) salários mínimos.

Foi observada uma distribuição homogênea quanto ao sexo na amostra, sendo $46,4 \%$ dos participantes do sexo masculino. A idade variou entre 10 e 17 anos, com média de 12,4 anos (desvio-padrão= 1,5) e mediana de 12,3 anos. Foi encontrado um percentual maior de adolescentes do sexo feminino na fase púbere (60,9\%), enquanto que a maioria dos participantes do sexo masculino $(73,5 \%)$ foi classificada como pré-púbere.

Observou-se um consumo médio de energia de $3.645 \mathrm{kcal}$ (IC: $3.509 \mathrm{kcal}-3.781 \mathrm{kcal}$ ), 
sem diferenças significantes segundo sexo (3.716kcal para o sexo masculino e $3.585 \mathrm{kcal}$ para o sexo feminino; $p=0,317$ ) e maturação sexual ( $3.551 \mathrm{kcal}$ para os pré-púberes e $3.671 \mathrm{kcal}$ para os púberes; $p=0,275)$. Os percentuais médios (e desvio-padrão) de energia provenientes de macronutrientes foram de 56,2 $(5,6) \%$ para carboidratos, 12,8 (2,3)\% para proteínas e de 33,5 $(4,8) \%$ para gorduras. Foi verificado reduzido consumo de frutas e de hortaliças, além de elevado consumo de doces (Tabela 1). Não foram observadas diferenças significantes quanto ao consumo dos grupos alimentares entre os sexos; porém, constatou-se que os adolescentes púberes apresentaram um consumo de doces significativamente mais elevado, em comparação com os pré-púberes.

Segundo a comparação estabelecida com as recomendações dietéticas, verificou-se que substancial parcela dos adolescentes entrevistados apresentou um consumo alimentar destoante do preconizado. Quanto à participação de macronu- trientes na dieta, destacou-se, principalmente, que a maioria dos participantes $(77,9 \%)$ apresentava dieta com um percentual de energia proveniente de gorduras acima dos limites preconizados para uma alimentação saudável (Figura 1). Nenhum adolescente apresentou teor de gordura da dieta abaixo do limite inferior recomendado ou teor de carboidratos acima do limite máximo recomendado. Cerca de 28,0\% dos adolescentes não atingiram a recomendação mínima de consumo de três porções de frutas e de três porções de hortaliças ao dia. A quase totalidade dos adolescentes (96,9\%) apresentou um consumo de doces acima de uma porção diária (Figura 2).

Foram identificados apenas 4,4\% dos participantes com baixo peso, enquanto que $21,0 \%$ da amostra apresentava algum grau de excesso de peso (Tabela 2). Constatou-se uma prevalência de $10,8 \%$ de obesidade entre os adolescentes. Foi observado que $1,8 \%$ da amostra tinha déficit de altura-para-idade, sendo 5 meninas e 2 meninos classificados nessa situação.

Tabela 1. Caracterização do consumo alimentar de adolescentes de escolas públicas de Piracicaba segundo sexo e maturação sexual. São Paulo, 2004.

\begin{tabular}{|c|c|c|c|c|c|c|c|c|}
\hline & \multicolumn{8}{|c|}{ Média (Intervalo de confiança de 95\%) } \\
\hline & \multicolumn{5}{|c|}{ Valor energético total (\%) } & \multicolumn{3}{|c|}{ Porções diárias } \\
\hline & Carboidratos & & Proteína & & Gordura & Frutas & Hortaliças & Doces \\
\hline Sexo masculino & $55,9(55,1-56,7)$ & 12,9 & $(12,6-13,2)$ & 33,7 & $(33,0-34,4)$ & $2,3(2,1-2,5)$ & $2,2(2,0-2,5)$ & $4,5(4,2-4,8)$ \\
\hline Sexo feminino & $56,5(55,7-57,3)$ & 12,7 & $(12,4-13,0)$ & 33,4 & $(32,7-34,0)$ & $2,4(2,1-2,6)$ & $2,5(2,3-2,8)$ & $4,4(4,1-4,7)$ \\
\hline Pré-púberes & $55,4 \quad(54,5-56,4)$ & 13,1 & $(12,6-13,5)$ & 34,2 & $(33,2-35,2)$ & $2,3(1,9-2,6)$ & $2,4(2,0-2,7)$ & $3,9(3,4-4,4)$ \\
\hline Púberes & $56,4(55,8-57,1)$ & 12,7 & $(12,5-13,0)$ & 33,3 & $(32,8-33,9)$ & $2,3(2,2-2,5)$ & $2,4(2,2-2,6)$ & $4,6(4,3-4,9)$ \\
\hline Total & $56,2 \quad(55,7-56,8)$ & 12,8 & $(12,6-13,0)$ & 33,5 & $(33,0-34,0)$ & $2,3(2,2-2,5)$ & $2,4(2,2-2,6)$ & $4,5(4,2-4,7)$ \\
\hline
\end{tabular}

Tabela 2. Distribuição do perfil antropométrico dos adolescentes de escolas públicas de Piracicaba segundo sexo e maturação sexual. São Paulo, 2004.

\begin{tabular}{|c|c|c|c|c|c|c|c|c|c|c|}
\hline \multirow{3}{*}{ Perfil antropométrico } & \multicolumn{4}{|c|}{ Sexo } & \multicolumn{4}{|c|}{ Maturação sexual } & \multirow{2}{*}{\multicolumn{2}{|c|}{ Total }} \\
\hline & \multicolumn{2}{|c|}{ Masculino } & \multicolumn{2}{|c|}{ Feminino } & \multicolumn{2}{|c|}{ Pré-púberes } & \multicolumn{2}{|c|}{ Púberes } & & \\
\hline & $\mathrm{n}$ & $\%$ & $\mathrm{n}$ & $\%$ & $n$ & $\%$ & $\mathrm{n}$ & $\%$ & $\mathrm{n}$ & $\%$ \\
\hline Baixo peso & 6 & 3,3 & 11 & 5,3 & 6 & 7,2 & 11 & 3,6 & 17 & 4,4 \\
\hline Eutrofia & 132 & 72,9 & 159 & 76,1 & 60 & 72,3 & 231 & 75,2 & 291 & 74,6 \\
\hline Sobrepeso & 18 & 10,0 & 22 & 10,5 & 8 & 9,6 & 32 & 10,4 & 40 & 10,2 \\
\hline Obesidade & 25 & 13,8 & 17 & 8,1 & 9 & 10,9 & 33 & 10,8 & 42 & 10,8 \\
\hline Total & 181 & 100,0 & 209 & 100,0 & 83 & 100,0 & 307 & 100,0 & 390 & 100,0 \\
\hline
\end{tabular}




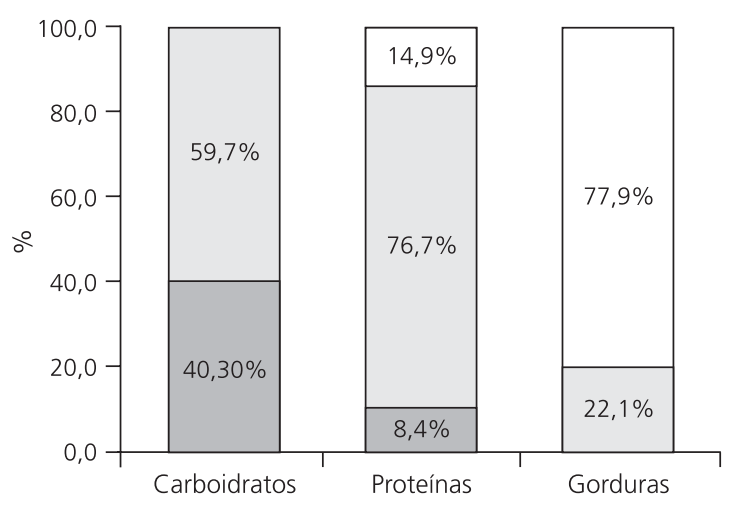

$\square$ Acima das recomendações $\square$ De acordo com as recomendações $\square$ Abaixo das recomendações

Figura 1. Distribuição percentual de adolescentes de escolas públicas de Piracicaba segundo as recomendações de distribuição de energia proveniente de macronutrientes na dieta, propostas pelo Guia alimentar para a população brasileira. São Paulo, 2004.

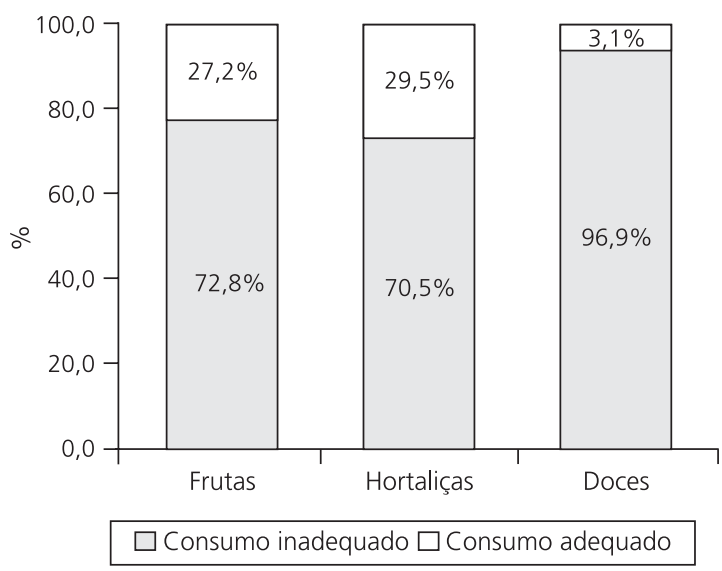

Figura 2. Distribuição percentual de adolescentes de escolas públicas de Piracicaba segundo as recomendações de consumo de frutas, hortaliças e doces, propostas pelo Guia alimentar para a população brasileira. São Paulo, 2004.

Não foi observada associação significante entre a classificação do perfil antropométrico e o sexo ou a maturação sexual. Também não foi verificada qualquer associação entre o consumo alimentar adequado e a ausência de excesso de peso entre os adolescentes do estudo.

\section{DISCUSS Ã O}

Os estudos brasileiros que investigaram o consumo alimentar de adolescentes utilizaram metodologias distintas, optando, freqüentemente, pela aplicação de Recordatórios de 24 horas. Porém, a aplicação de um único Recordatório de 24 horas permite apenas avaliar a dieta atual do indivíduo e não a habitual. Por outro lado, os Questionários de Freqüência Alimentar são de grande utilidade quando adotados em estudos epidemiológicos, pois permitem o conhecimento do consumo alimentar habitual de uma população e possibilitam a classificação dos indivíduos segundo o grau de exposição ${ }^{16}$.

No Brasil, inexistem pesquisas de base populacional representativas do País que tenham utilizado o Questionário de Freqüência Alimentar do tipo semi-quantitativo. Os dados provenientes de levantamentos internacionais, como os National Health and Nutrition Examination Surveys (NHANES), realizados nos Estados Unidos, se distanciam dos encontrados no presente estudo, notadamente pela diferença na metodologia adotada: o Recordatório de 24 horas $^{17}$. Pesquisas brasileiras que utilizaram esse inquérito alimentar em estudos pontuais, também revelaram dados de consumo inferiores ao encontrado neste estudo ${ }^{18,19}$.

Em estudo realizado na Noruega, envolvendo 1.564 adolescentes, verificou-se, por meio de um questionário de freqüência alimentar semiquantitativo, que o consumo de energia médio foi de $3.776 \mathrm{kcal}$ entre os meninos e $2.366 \mathrm{kcal}$ entre as meninas ${ }^{20}$. Os dados de consumo de energia se revelaram muito próximos aos encontrados neste trabalho.

Escassos estudos nacionais referem a adoção de questionários de freqüência alimentar validados para a população em estudo, principalmente envolvendo adolescentes. No município de Niterói, no Rio de Janeiro, foi avaliado o consumo alimentar de 391 adolescentes de uma escola privada, por meio de um questionário de freqüência alimentar semi-quantitativo ${ }^{21}$. $\mathrm{O}$ instrumento 
havia sido previamente validado para adultos e modificado para sua aplicação entre adolescentes, com a inclusão de alimentos e a alteração do tamanho das porções. Verificou-se que, em média, os adolescentes eutróficos e com sobrepeso do sexo masculino consumiam $3.188 \mathrm{kcal}$ e $2.819 \mathrm{kcal}$, respectivamente. As meninas apresentaram um consumo médio de energia de $2.462 \mathrm{kcal}$, entre as eutróficas, e $2.209 \mathrm{kcal}$ entre aquelas com sobrepeso.

Andrade et al. ${ }^{22}$ estimaram o consumo alimentar de 387 adolescentes por meio de um questionário de freqüência semi-quantitativo no município do Rio de Janeiro. O objetivo do estudo foi estabelecer comparações entre o consumo alimentar de adolescentes com e sem sobrepeso, que resultou ser semelhante em ambos os grupos. Foi observado que os meninos (com e sem sobrepeso) apresentavam valores de consumo médios de energia de $2.890 \mathrm{kcal}$ e $2.697 \mathrm{kcal}$, respectivamente. Entre as meninas com sobrepeso, identificou-se uma ingestão de $2.848 \mathrm{kcal}$, enquanto que aquelas sem sobrepeso revelaram um consumo de $2.920 \mathrm{kcal}$.

Sugere-se que os dados deste estudo possam estar superestimados, devido a alguns fatores relacionados ao uso do Questionário de Freqüência Alimentar, como a apresentação de porções padronizadas, a presença de vários alimentos agrupados no mesmo item e o número de itens registrados no instrumento. Tais pontos indicam a presença de um erro sistemático de superestimação no questionário de freqüência alimentar adotado $^{23}$. Por outro lado, cabe ressaltar que foi adotado um inquérito validado para adolescentes de São Paulo, que permite a avaliação do consumo alimentar nos últimos 6 meses ${ }^{12}$. A validação de um instrumento significa que o mesmo mede o que se propõe a medir $^{16}$. A utilização de um inquérito alimentar validado fez parte do cuidadoso procedimento metodológico a que foi submetido este estudo, garantindo maior confiabilidade e precisão das informações.

Destaca-se ainda que estudos dietéticos envolvendo crianças e adolescentes incluem outras limitações, como a menor habilidade cognitiva de recordar e registrar o consumo alimentar e o tamanho das porções, a falta de conhecimento de determinadas preparações e o valor social atribuído a alguns alimentos. Além disso, sabe-se que os alimentos preferidos pelos entrevistados tendem a ser lembrados com maior facilidade, havendo superestimação do tamanho das porções. Por outro lado, alimentos que não são do agrado do indivíduo tendem a ser esquecidos ou ser atribuída uma reduzida freqüência de consumo aos mesmos ${ }^{23,24}$.

Quanto à distribuição dos macronutrientes na alimentação, foi considerado alarmante, principalmente, o percentual de adolescentes que apresentaram alto consumo de gorduras (77,9\%), com percentual de participação desse nutriente acima de 30,0\% do valor energético total. Foram encontrados na literatura resultados semelhantes em relação à expressiva proporção de adolescentes que consomem teores elevados de gorduras na dieta. Em diversos locais do mundo, como nos Estados Unidos ${ }^{17}$, na Noruega ${ }^{20}$ e na Finlândia ${ }^{25}$, foram identificados elevados teores de gordura na dieta dos adolescentes. Quadro semelhante é observado entre adolescentes de países do sul da Europa, como Espanha, Grécia, Itália e Portugal, revelando que os aspectos saudáveis característicos da Dieta Mediterrânea, possivelmente, estão sendo abandonados ${ }^{26}$.

Estudos brasileiros também têm demonstrado a participação elevada das gorduras na dieta dos adolescentes. Entre os escolares avaliados por Albano \& Souza ${ }^{18}$, o percentual de energia proveniente de gorduras foi de $32,2 \%$ para os meninos e $29,9 \%$ para as meninas. No estudo de Garcia et al. ${ }^{19}$, observou-se uma participação média de $31,3 \%$ de gorduras na dieta dos adolescentes, sendo que $53,3 \%$ dos meninos e $41,0 \%$ das meninas apresentaram um consumo elevado de colesterol.

Conforme o esperado, foi encontrado um consumo reduzido de frutas e hortaliças entre os adolescentes deste estudo. Estudos realizados na Suécia demonstraram um baixo consumo desses 
grupos alimentares notadamente na adolescência, sendo que somente $40 \%$ dos jovens com 15 anos de idade consumiam frutas e verduras diariamente ${ }^{25}$. Na Austrália, também foi observado baixo consumo de frutas e verduras entre 1.656 crianças e adolescentes com idade de 5 a 15 anos, tanto no ambiente escolar como fora deste ${ }^{27}$.

Nos Estados Unidos, os dados dos Levantamentos Contínuos de Consumo Alimentar de Indivíduos (Continuing Survey of Food Intake by Individuals - CSF), realizados em 1989-1991 (CSFI) e em 1994-1996 (CSFII), mostraram que há uma ligeira tendência de aumento do consumo de frutas e verduras no país ${ }^{28}$. Porém, observou-se que o consumo desses alimentos na população alcançava apenas o número mínimo de porções preconizadas. Segundo o CFSII, as crianças e adolescentes avaliados $(n=5.144)$ consumiam diariamente, em média, 1,6 porções de frutas e 2,7 porções de verduras.

Em estudo nacional, realizado com 185 adolescentes recém-ingressos em uma universidade pública, verificou-se que $75,1 \%$ dos entrevistados registraram o consumo de frutas quatro vezes ou menos na semana ${ }^{29}$. As hortaliças foram as mais citadas entre as rejeições alimentares, sendo que $79,5 \%$ dos universitários citaram, pelo menos, um alimento desse grupo. Garcia et al. ${ }^{19}$ também constataram o reduzido consumo de frutas e hortaliças entre adolescentes brasileiros.

Outro problema constantemente relatado na literatura refere-se ao expressivo consumo de açúcares e doces na alimentação dos adolescentes, situação observada entre os entrevistados. Segundo pesquisa realizada entre adolescentes dinamarqueses, constatou-se que $75 \%$ dos entrevistados consumiam açúcares de adição acima do limite recomendado pelas autoridades sanitárias dos países nórdicos, de 10\% de energia proveniente desse componente alimentar ${ }^{25}$. Estudos realizados na Noruega, na Espanha e também no Brasil, verificaram a presença de um quadro semelhante em relação ao consumo de doces entre adolescentes ${ }^{19,26,29}$.
A alimentação dos participantes deste estudo não apresentou associação com a expressiva prevalência de excesso de peso encontrada. Contudo, é necessário cautela para a interpretação de tais achados. Em primeiro lugar, ressalta-se que estudos transversais não permitem o estabelecimento de uma relação de causa e efeito dessa magnitude. Sugere-se que estudos com delineamento longitudinal investiguem em maior profundidade a relação entre a dieta inadequada e os possíves reflexos no perfil antropométrico dos adolescentes. Da mesma forma, seria interessante investigar outros fatores que podem contribuir para o excesso de peso na amostra, como o sedentarismo, considerando que essa variável não foi contemplada no presente estudo. Cabe destacar ainda que, apesar de não terem sido detectadas associações da alimentação com a antropometria, existe a possibilidade de ocorrerem deficiências de micronutrientes na população estudada como outro possível efeito deletério do desequilíbrio alimentar, principalmente pelo baixo consumo de frutas e hortaliças.

Sabe-se que o elevado consumo energético, decorrente da participação de alimentos ricos em gordura e açúcar, em porções cada vez maiores, tem sido freqüentemente associado ao desenvolvimento da obesidade ${ }^{9,30}$. Os dados de prevalência de excesso de peso entre os adolescentes de Piracicaba são extremamente preocupantes, mas condizentes com o panorama mundial observado. Destaca-se que o excesso de peso afeta percentuais elevados de adolescentes em diversas regiões, como no Reino Unido (21\%), Espanha (21\%), Grécia (22\%) e llha de Chipre (23\%). Nos Estados Unidos, uma em cada quatro crianças tem excesso de peso ${ }^{31}$.

No Brasil, estudos isolados também têm detectado a elevada prevalência de excesso de peso na adolescência e mostram dados semelhantes aos encontrados na presente pesquisa ${ }^{22,32}$. O mesmo quadro é observado entre os levantamentos nacionais realizados com adolescentes. Dados recentes mostram que, nas últimas décadas, tem sido observado no Brasil um aumento dos referidos percentuais nessa faixa etária, em 
ambos os sexos ${ }^{33}$. Verificou-se que o excesso de peso dos adolescentes brasileiros atingia 18,0\% dos meninos e $15,4 \%$ das meninas, segundo avaliação da última Pesquisa de Orçamentos Familiares (POF), conduzida em 2002-2003, sendo que dados do ENDEF, de 1974-1975, mostravam valores de $3,9 \%$ de excesso de peso entre os meninos e $7,5 \%$ entre as meninas.

Por outro lado, neste estudo foi encontrado um percentual reduzido de adolescentes com déficit de altura-para-idade (1,8\%) em comparação com os achados da literatura. Entre adolescentes de escolas públicas do município de Teixeira de Freitas, na Bahia, identificou-se uma prevalência de $25,0 \%$ de déficit de crescimento ${ }^{34}$. Da mesma forma, segundo a POF de 2002-2003, cerca de $10,0 \%$ dos adolescentes brasileiros apresentavam déficits de altura-para-idade, sendo o problema mais freqüente entre os meninos $(11,3 \%)$ do que entre as meninas $(8,3 \%)^{33}$, ao contrário do observado no presente estudo. Tais dados sugerem que a maioria dos adolescentes entrevistados apresenta um crescimento linear adequado, indicando boas condições de saúde e nutrição na infância de forma geral, principalmente nos primeiros anos de vida.

\section{O N CLUS Ã O}

O consumo alimentar e o perfil antropométrico dos adolescentes da rede pública de ensino de Piracicaba estão de acordo com o processo de transição nutricional que ocorre no Brasil. Considerando o desequilíbrio alimentar e a elevada prevalência de excesso de peso observados entre os adolescentes, o grupo avaliado deve ser alvo imediato de intervenções, entre elas, as ações educativas que estimulem a adoção de uma alimentação saudável desde o nascimento, firmando-se na adolescência para que seja mantida na vida adulta.

\section{COLABORADORES}

N. TORAL participou de todas as etapas do estudo e redigiu a versão inicial do artigo, que se refere a parte de sua dissertação de Mestrado, intitulada "Estágios de Mudança de Comportamento e sua relação com o consumo alimentar de adolescentes", defendida em março de 2006, na Faculdade de Saúde Pública da Universidade de São Paulo. B. SLATER contribuiu com análises estatísticas e redação do artigo. M.V. SILVA participou da revisão do artigo e da coordenação do projeto que envolveu o estudo.

\section{A GRADECIMENTOS}

A autora Natacha Toral agradece à Fundação de Amparo à Pesquisa do Estado de São Paulo (FAPESP) pela bolsa de estudos concedida ( $n^{\circ}$ 04/03871-3) e pelos recursos financeiros oferecidos ( $n^{\circ} 02 / 9521-9$ ).

\section{REFERÊ NCIAS}

1. Eisenstein E, Coelho KSC, Coelho SC, Coelho MASC. Nutrição na adolescência. J Pediatr. (Rio J) 2000; 76(Supl 3):S263-S74.

2. Fisberg M, Bandeira CRS, Bonilha EA, Halpern G, Hirschbruch MD. Hábitos alimentares na adolescência. Pediatr Mod. 2000; 36(11):724-34.

3. Pedrinola F. Nutrição e transtornos alimentares na adolescência. Pediatr Mod. 2002, 38(8):377-80.

4. Fisberg M. Atualização em obesidade na infância e adolescência. São Paulo: Atheneu; 2004.

5. Batista Filho $M$, Rissin A. A transição nutricional no Brasil: tendências regionais e temporais. Cad Saúde Pública. 2003; 19(Supl 1):S181-S91.

6. Veiga GV, Cunha AS, Sichieri R. Trends in overweight among adolescents living in the poorest and richest regions of Brazil. Am J Public Health. 2004; 94(9):1544-8.

7. World Health Organization. Diet, nutrition and the prevention of chronic diseases. Geneva; 2003. Technical Report Series, 916.

8. Instituto Brasileiro de Geografia e Estatística. Censo demográfico: 2000 [online]. Rio de Janeiro; 2000 [acesso em 2 abr 2005]. Disponível em: http: //www.ibge.gov.br/censo/default.php

9. Ludwig DS, Peterson KE, Gortmaker SL. Relation between consumption of sugar-sweetened drinks and childhood obesity: a prospective, observational analysis. Lancet. 2001; 357(9255):505-8.

10. Tanner JM. Growth at adolescence. 2nd ed. Oxford: Blackwell Scientific Publications; 1962. 
11. World Health Organization. Physical status: the use and interpretation of antropometry. Geneva; 1995. Technical Report Series, 854.

12. Slater B, Fisberg RM, Philippi ST, Latorre MRDO. Validation of a semi-quantitative adolescents food frequency questionnaire applied at a public school in São Paulo, Brazil. Eur J Clin Nutr. 2003; 57(5): 629-35.

13. Brasil. Ministério da Saúde. Secretaria de Atenção à Saúde. Coordenação-Geral da Política de Alimentação e Nutrição. Guia alimentar para a população brasileira: promovendo a alimentação saudável. Série A. Normas e manuais técnicos. Brasília; 2005.

14. Centers for Disease Control and Prevention. National Center for Health Statistics. CDC Growth Charts 2000. United States [cited 2005 Oct 21]. Available from: http://www.cdc.gov/growthcharts

15. National Center for Health Statistics. Growth curves for children birth - 18 years. Washington (DC): US Government Printing Office; 1977. Vital and Health Statistics Series, 11, 165.

16. Fisberg RM, Slater B, Marchioni DML, Martini LA. Inquéritos alimentares: métodos e bases científicos. Barueri: Manole; 2005.

17. Troiano RP, Briefel RR, Carroll MD, Bialostosky K. Energy and fat intakes of children and adolescents in the United States: data from the National Health and Nutrition Examination Surveys. Am J Clin Nutr. 2000; 72(Suppl):1343S-53S.

18. Albano RD, Souza SB. Ingestão de energia e nutrientes por adolescentes de uma escola pública. J Pediatr. (Rio J) 2001; 77(6):512-6.

19. Garcia GCB, Gambardella AMD, Frutuoso MFP. Estado nutricional e consumo alimentar de adolescentes de um centro de juventude da cidade de São Paulo. Rev Nutr. 2003; 16(1):41-50.

20. Andersen LF, Nes M, Sandstad B, Bjørneboe G-EA, Drevon CA. Dietary intake among Norwegian adolescents. Eur J Clin Nutr. 1995; 49(8):555-64.

21. Fonseca VM, Sichieri R, Veiga GV. Fatores associados à obesidade em adolescentes. Rev Saúde Pública. 1998; 32(6):541-9.

22. Andrade RG, Pereira RA, Sichieri R. Consumo alimentar de adolescentes com e sem sobrepeso do Município do Rio de Janeiro. Cad Saúde Pública. 2003; 19(5):1485-95.

23. Slater B. Desenvolvimento e validação de um questionário semi-quantitativo de freqüência alimentar para adolescentes [tese]. São Paulo: Faculdade de Saúde Pública, Universidade de São Paulo; 2001.

24. Drewnowski A. Diet image: a new perspective on the food-frequency questionnaire. Nutr Rev. 2001; 59(11):370-2.

25. Samuelson $G$. Dietary habits and nutritional status in adolescents over Europe. An overview of current studies in the Nordic countries. Eur J Clin Nutr. 2000; 54(Suppl 1):S21-S8.

26. Cruz JAA. Dietary habits and nutritional status in adolescents over Europe - Southern Europe. Eur J Clin Nutr. 2000; 54(Suppl 1):S29-S35.

27. Bell AC, Swinburn BA. What are the key food groups to target for preventing obesity and improving nutrition in schools? Eur J Clin Nutr. 2004; 58(2):258-63.

28. Krebs-Smith SM, Kantor LS. Choose a variety of fruits and vegetables daily: understanding the complexities. J Nutr. 2001; 131(Suppl):487S-501S.

29. Vieira VCR, Priore SE, Ribeiro SMR, Franceschini SCC, Almeida LP. Perfil socioeconômico, nutricional e de saúde de adolescentes recém-ingressos em uma universidade pública brasileira. Rev Nutr. 2002; 15(3):273-82.

30. Nielsen SJ, Popkin BM. Patterns and trends in food portion sizes, 1977-1998. JAMA. 2003; 289(4): 450-3.

31. Popkin BM, Gordon-Larsen P. The nutrition transition: worldwide obesity dynamics and their determinants. Int J Obes. 2004; 28(Suppl 3):S2-S9.

32. Carvalho CMRG, Nogueira AMT, Teles JBM, Paz SMR, Sousa RML. Consumo alimentar de adolescentes matriculados em um colégio particular de Teresina, Piauí, Brasil. Rev Nutr. 2001; 14(2):85-93.

33. Instituto Brasileiro de Geografia e Estatística. Pesquisa de Orçamentos Familiares (POF) 2002-2003: antropometria e análise do estado nutricional de crianças e adolescentes no Brasil. Rio de Janeiro; 2006.

34. Santos JS, Costa MCO, Nascimento Sobrinho CL, Silva MCM, Souza KEP, Melo BO. Perfil antropométrico e consumo alimentar de adolescentes de Teixeira de Freitas - Bahia. Rev Nutr. 2005; 18(5): 623-32.

Recebido em: 24/3/2006

Versão final reapresentada em: 13/4/2007 Aprovado em: 22/5/2007 
